\title{
Effects of different probiotics on the gut microbiome and metabolites in the serum and caecum of weaning piglets
}

\author{
X. Chen ${ }^{1}$, Y.Z. Liu ${ }^{1}$, N. Demelash ${ }^{1}$, J.N. Dong ${ }^{1}$, J. Xiao ${ }^{2}$, W. Zhao ${ }^{1}$, M. Lang ${ }^{1}$, Z.Y. Han ${ }^{1}$,

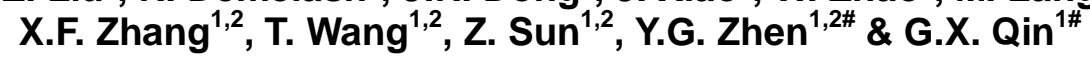 \\ ${ }^{1}$ College of Animal Science and Technology, Jilin Agricultural University, Changchun, 130118, P.R. China; \\ ${ }^{2}$ JLAU-Borui Dairy Science and Technology R\&D Centre of Jilin Agricultural University, Changchun 130118, P.R. China
}

(Received 14 December 2018; Accepted 18 April 2019; First published online 2 June 2019)

\author{
Copyright resides with the authors in terms of the Creative Commons Attribution 4.0 South African License. \\ See: http://creativecommons.org/licenses/by/4.0/za \\ Condition of use: The user may copy, distribute, transmit and adapt the work, but must recognize the authors and \\ the South African Journal of Animal Science.
}

\begin{abstract}
The objective of the study was to determine the effects of antibiotics, yeast culture (YC), and Lactobacillus culture (LC) on the gut microbiome and metabolites in the serum and caecum of weaning piglets. Twenty-four weaning piglets were divided into four treatment groups: control, antibiotic (1\% chlortetracycline), $1.8 \%$ yeast culture (YC), and 1.6\% Lactobacillus culture groups (LC). Each group had six replicated pens with one pig per pen. Feed and water were available ad libitum. Dietary supplementation with antibiotics, YC and LC increased the abundance of phylum, Firmicutes, and decreased the abundance of phylum, Proteobacteria. Beneficial bacteria such as Lactobacillus and Megasphaera in YC and LC groups increased, whereas the proportion of Shigella was decreased. Genera Alloprevotella and Lachnospira were biomarkers in the control and antibiotic groups, respectively. Phylum, Bacteroidetes, and genus, Collinsella, were biomarkers in the YC group, and Mitsuokella, Anaerotruncus, Syntrophococcus and Sharpea were biomarkers in the LC group. Dietary supplementation with different probiotics changed the serum and caecum metabolite profiles too. Antibiotic supplementation increased the levels of D-mannose, D-glucose, and hexadecanoic acid in the serum, and the levels of myo-inositol, D-mannose and benzenepropanoic acid in the caecum. LC increased the concentrations of D-mannose, cis-9-hexadecenoic acid and heptadecanoic acid in caecum compared with the control group. YC and LC supplementation in the weaning diet could improve the abundance of beneficial bacteria by changing the concentrations of some metabolites in the serum and caecum. Therefore, dietary supplementation with YC or LC could be used as additives instead of antibiotics in weaning piglets.
\end{abstract}

Keywords: antibiotic; lactobacillus culture; yeast culture; high-throughput sequencing; gas chromatography mass spectrometry

\#Corresponding authors: nickzhen@263.net; qgx@jlau.edu.cn

\section{Introduction}

Weaning in pigs is a critical but complex step, which involves a significant amount of stress, and interferes acutely with feed intake, intestinal metabolism, the immune system and the gastrointestinal microbiome (Williams et al., 1997; Torrallardona et al., 2007; Zhou et al., 2016; Niederwerder et al., 2017). Although some studies have indicated that low doses of antibiotics can improve the growth of animals, there are long-term negative effects on animal and human health (Sekirov et al., 2008; Heo et al., 2013). Since 2006 the European Union has banned the use of antibiotics as growth promoters in animal feed to prevent risk to human beings from antibiotic residues in animals (Castanon et al., 2007). A large number of studies have paid close attention to the use of probiotics as additives to improve pig performance and health without antibiotics. Previous studies have shown that dietary supplementation with a Lactobacillus culture (LC) improves performance and maintains the intestinal health of pigs by increasing the amount of faecal Lactobacillus and decreasing Escherichia coli (Giang et al., 2010; Lan et al., 2016). According to Weedman et al. (2011), Xiong et al. (2015) and Jiang et al. (2015), yeast culture (YC) can replace antibiotics in the diets of piglets to improve their intestinal functioning and serum biochemical variables. 
The gastrointestinal microbiome plays an important role in the health of the host by increasing the colonization of beneficial bacteria that promote gut maturation and prevent invading pathogens (Lan et al., 2005; Fouhse et al., 2016). Metabolomics is an important tool for understanding biological systems and has been used successfully to study changes in serum metabolites when L-arginine supplementation is given to growing pigs (He et al., 2009; Dona et al., 2016). Currently, high throughput and gas chromatography mass spectrometry (GC/MS) and liquid chromatography mass spectrometry (LC/MS) methods are preferred for studying changes in gastrointestinal microbiomes and metabolites. However, research on the effects of antibiotics, YC and LC on the gut microbiome, serum metabolites and gut metabolites is still limited. Therefore, in this study the authors investigated YC and LC fermented in the laboratory as antibiotic alternatives in livestock by studying their effects on the gut microbiome and metabolites in the serum and caecum of newly weaned piglets.

\section{Materials and Methods}

Yeast culture (YC) and LC were both developed in the JLAU-Borui Dairy Science and Technology R\&D Centre of Jilin Agricultural University (Changchun, P.R. China). They were produced by fermentation of Saccharomyces cerevisiae and Lactobacillus plantarum, respectively. The cultures were aerobically incubated in a $10 \mathrm{~L}$ fermenter and then transferred into a $50 \mathrm{~L}$ fermenter for 24 hours. Papain was added to induce yeast cell wall breakage, and the fermentation was continued for 36 hours. The yeast cell wallbreakage rate was $55.48 \%$, and the dry weights of $Y C$ and LC were $150.76 \mathrm{~g} / \mathrm{L}$ and $99.44 \mathrm{~g} / \mathrm{L}$, respectively. YC and LC are liquids that contain yeast extracellular metabolites, fermented medium, broken cell walls, cellular contents and dead bacteria.

A total of 24 piglets (General No. 1, barrows) that had been weaned at an average age of 28 days with an initial body weight of $10.5 \pm 0.71 \mathrm{~kg}$ were purchased from the Pig Breeding Farm of Jilin University (Changchun, P.R. China). They were divided into four treatment groups: i) control group, ii) antibiotic group (1\% chlortetracycline) (Jinhe Biotechnology Co. Ltd., China), iii) $1.8 \%$ yeast culture group (YC), and iv) $1.6 \%$ lactobacillus culture group (LC). Each treatment had six pens with one piglet per pen. Feed and water were available ad libitum during the four weeks of the feeding period. All animals were housed in smallholder styled pens. All diets were formulated according to the nutrient requirements of NRC (2012), as is indicated in Table 1.

Table 1 Ingredients and chemical compositions of the basal diets for weaning piglets

\begin{tabular}{lc|cc}
\hline Ingredient & Content, \% & Nutritive value & Content \\
\hline Corn & 65.65 & Metabolizable energy, MJ/kg & 13.60 \\
Wheat bran & 1.00 & Crude protein, \% & 19.00 \\
Whey powder & 3.00 & Calcium, \% & 0.74 \\
Casein & 2.05 & Total phosphorus, \% & 0.58 \\
Fish meal & 8.54 & Lysine, \% & 1.16 \\
Meat meal & 0.90 & & \\
Soybean meal & 14.00 & & \\
Salt & 0.135 & & \\
Limestone & 0.70 & & \\
Sucrose & 3.00 & & \\
lysine & 0.04 & & \\
Additive & 1.00 & & \\
Total & 100.00 & & \\
\end{tabular}

Supplied per kilogram of diet on DM basis: vitamin A: 28500 IU; vitamin $D_{3}: 6000$ IU; vitamin E: 67.5 IU; vitamin $\mathrm{K}_{3}: 7.5$ mg; vitamin $B_{1}: 7.5 \mathrm{mg}$; vitamin $B_{2}: 15$ mg; vitamin $B_{6}: 9$ mg; vitamin $B_{12}: 0.075$ mg; folic acid: 3 mg; nicotinic acid: 75 mg; biotin: $0.375 \mathrm{mg}$; pantothenic acid: $37.5 \mathrm{mg}$; antioxygen: $0.15 \mathrm{mg}$; choline chloride: $100 \mathrm{mg}$; Fe: $150 \mathrm{~g}$; Cu: $150 \mathrm{mg}$; Mn: 80 mg; Zn: 120 mg; l: 0.3 mg; Se: 0.3 mg; Co: $1 \mathrm{mg}$. 
The animals were slaughtered on day 28. Blood samples were collected from the hepatic portal veins, and serum was obtained by centrifugation at $3500 \times \mathrm{g}$, at a temperature of $4{ }^{\circ} \mathrm{C}$ for $10 \mathrm{~min}$ and stored at $-80{ }^{\circ} \mathrm{C}$ until needed for the GC/MS analysis. One side of each caecum (digesta) was tied using string and stored at $-20^{\circ} \mathrm{C}$ for DNA extraction, and the remaining part was stored at $-80^{\circ} \mathrm{C}$ until GC/MS analysis.

The DNA was isolated by the FastDNA ${ }^{\circledR}$ Spin Kit for Soil (MP Biotechnology, Santa Ana, CA, USA), according to the manufacturer's protocol. The DNA samples were sent to Biomarker Technologies Co, Ltd. (China) for polymerase chain reaction (PCR) amplification and high-throughput sequencing with the Illumina Hiseq method. The bacterial V3-V4 regions of the 16S rRNA genes were amplified with primers 5'-ACTCCTACGGGAGGCAGCA-3' (Huse et al., 2008) and 5'-GGACTACHVGGGTWTCTAAT-3' (Caporaso et al., 2012).

The serum samples $(100 \mu \mathrm{L})$ were mixed with $300 \mu \mathrm{L}$ methyl alcohol : chloroform (3: 1). After 10 min incubation at $-20^{\circ} \mathrm{C}$, the mixed liquor was centrifuged at $12000 \times g$ at $4{ }^{\circ} \mathrm{C}$ for $10 \mathrm{~min}$, and then $300 \mu \mathrm{L}$ of supernatant was transferred into test tubes. Samples were dried using nitrogen gas (caecum samples were dried using a freeze drier for 24 hours). The dried serum samples $(0.05 \mathrm{~g})$ were stored in $1.5 \mathrm{~mL}$ tubes. Mixed liquid (methoxyamine hydrochloride and pyridine) of $60 \mu \mathrm{L}(20 \mathrm{mg} / \mathrm{mL})$ was added to the serum samples (100 $\mu \mathrm{L}$ for caecum samples) and vortexed vigorously for $1 \mathrm{~min}$, followed by heating in a water bath at $37^{\circ} \mathrm{C}$ for $120 \mathrm{~min}(90 \mathrm{~min}$ for caecum samples). Subsequently, $60 \mu \mathrm{L}$ bis(trimethylsilyl)-trifluoroacetamide (BSTFA) with $1 \%$ trimethylchlorosilane (TMCS) was added to the serum samples and heated at $37^{\circ} \mathrm{C}$ for 60 min (200 $\mu$ L BSTFA with $1 \%$ TMCS added to the caecum samples) and heated at $70^{\circ} \mathrm{C}$ for $60 \mathrm{~min}$. After 10 $\mathrm{min}$, the samples were centrifuged at $13000 \times \mathrm{g}$ at $4{ }^{\circ} \mathrm{C}$ for $5 \mathrm{~min}$, and then $50 \mu \mathrm{L}$ supernatant of each sample was transferred into GC vials.

The analysis of each serum sample $(1 \mu \mathrm{L})$ was performed on an Agilent $7890 / 5975 \mathrm{C}$ system equipped with a $30.0 \mathrm{~m} \times 0.25 \mathrm{~mm}$ i.d. fused silica capillary column with a $0.25-\mu \mathrm{m}$ HP-5MS stationary phase (Agilent, Shanghai, China). Helium was used as the carrier at a constant flow rate of $1.0 \mathrm{~mL} / \mathrm{min}$. The temperature of the column was maintained at $70^{\circ} \mathrm{C}$ for $4 \mathrm{~min}$, increased to $100{ }^{\circ} \mathrm{C}$ at a rate of $4{ }^{\circ} \mathrm{C} / \mathrm{min}$ and maintained for 5 min, increased to $150^{\circ} \mathrm{C}$ at a rate of $10^{\circ} \mathrm{C} / \mathrm{min}$ and maintained for $10 \mathrm{~min}$, then increased to $240{ }^{\circ} \mathrm{C}$ at a rate of $5{ }^{\circ} \mathrm{C} / \mathrm{min}$ and maintained for $8 \mathrm{~min}$, and finally increased to $270^{\circ} \mathrm{C}$ at a rate of $8^{\circ} \mathrm{C} / \mathrm{min}$ and maintained for $1 \mathrm{~min}$ (increased to $270{ }^{\circ} \mathrm{C}$ at a rate of $10^{\circ} \mathrm{C} / \mathrm{min}$ and maintained for $5 \mathrm{~min}$ for caecum samples). The temperatures of injection, quadrupole, and ion source were $280^{\circ} \mathrm{C}, 230^{\circ} \mathrm{C}$ and $150{ }^{\circ} \mathrm{C}$, respectively. The mass spectra were acquired from $20 \sim 800 \mathrm{~m} / \mathrm{z}$.

The raw paired-end reads of the original DNA fragments were merged using FLASH, and the minimal overlap was $10 \mathrm{bp}$. To obtain clean tags, the raw tags were filtered with Trimmomatic v0.33 (Magoč \& Salzberg, 2011; Bolger et al., 2014). Chimeras of the clean tags were removed using UCHIME (Edgar et al., 2011). All of the effective reads from each sample were aligned using the UCLUST (Edgar, 2010) and clustered into operational taxonomic units (OTUs) at $97 \%$ sequence identity. The microbial sequencing and metabolic profiles data were processed by QIIME and SIMCA software. The variable importance in the projection $(\mathrm{VIP}>1)$ values and Student's $t$-test $(P<0.05)$ were compared to find differences among groups.

\section{Results and Discussion}

A total of 1552343 raw tags were checked by high-throughput pyrosequencing. After filtering, 1515749 clean tags with an average of 63156 were obtained. A total of 704 OTUs were obtained among the 24 samples (Figure 1). OTU-Venn showed that 581 OTUs were common species and only the control group and the LC group had 1 and 7 specific species, respectively. The main research interest of the gut microbiome is to determine whether specific microbiota can be delineated for different groups. To identify specific microbial candidates that are present in various breeding groups, LEFse (Ida score $>4$ ) was used to determine specific biomarkers that belonged to different taxa (Figure 2). Genera Mitsuokella, Anaerotruncus, Syntrophococcus and Sharpea (phylum Firmicutes) were higher in the LC group. Phylum, Bacteroidetes, and genus, Collinsella (phylum Actinobacteria), were higher in the YC group. Genera Lachnospira and Alloprevotella were biomarkers in the antibiotic and control groups, respectively. 


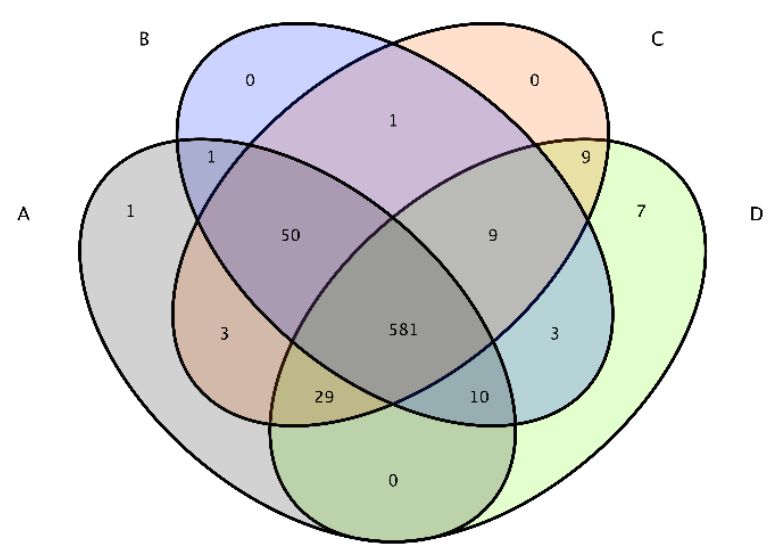

Figure 1 OTU-Venn of 704 OTUs calculated at $97 \%$ dissimilarity

$\mathrm{A}=$ control group; $\mathrm{B}=$ antibiotic group; $\mathrm{C}=$ yeast culture group; and $\mathrm{D}=$ lactobacillus culture group

Cladogram

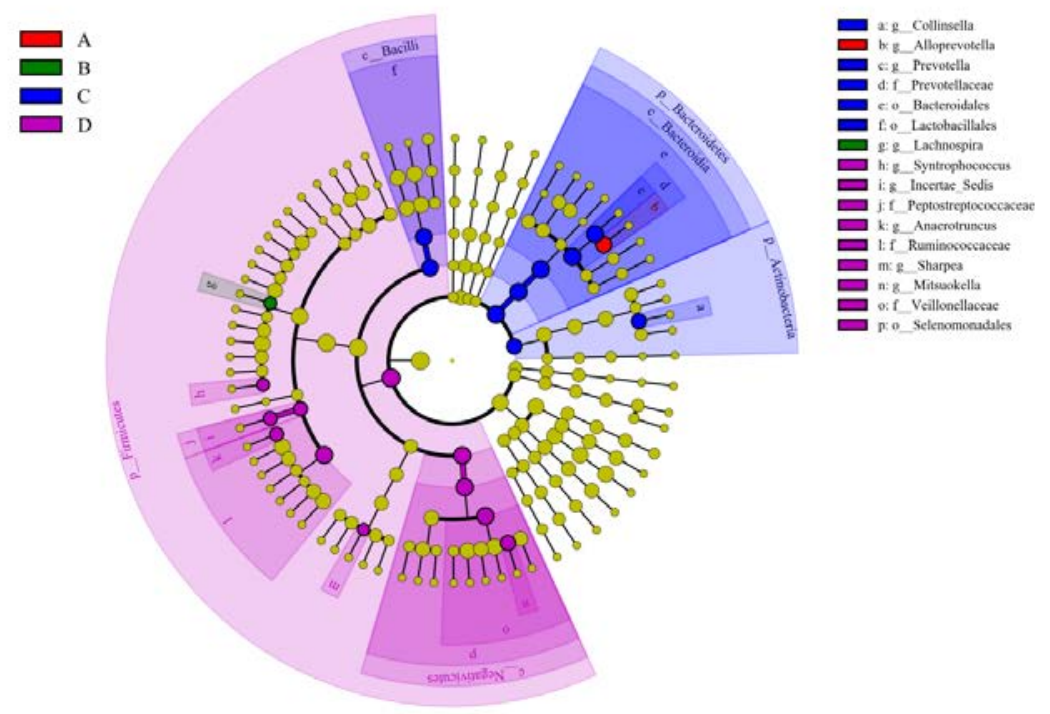

Figure 2 Evolutionary tree of caecal bacterial communities for each sample

$\mathrm{A}=$ control group; $\mathrm{B}=$ antibiotic group; $\mathrm{C}=$ yeast culture group; and $\mathrm{D}=$ lactobacillus culture group

All groups harboured diverse lineages of bacterial phyla (Figure 3). A total of 10 phyla were detected in all groups. The predominant phylum was Firmicutes, followed by phyla Bacteroidetes and Proteobacteria. The proportions of Firmicutes in groups A, B, C and D were $49.4 \%, 63.3 \%, 55.5 \%$ and $86.4 \%$, respectively. The ratios of phyla Firmicutes to Bacteroides were $1.44 \%, 2.15 \%, 1.61 \%$ and $11.06 \%$, respectively. The phylum, Proteobacteria, in the control group was represented in higher abundance than in the other groups. The concentration of phylum, Cyanobacteria, was more than $1 \%$ only in the antibiotic group. Phylum, Actinobacteria, in the YC group was 3.38\%, but was less than $1 \%$ in the other groups. The abundances of phyla Candidate_division_TM7, Chlamydiae, Fibrobacteres, Spirochaetae and Tenericutes in all groups were $<1 \%$.

The caecum sequencing of the weaning piglets detected 89, 89, 91 and 93 genera from the four groups, respectively. The genera with over $1 \%$ abundance differed among the groups (Table 2). The genus, Prevotella, was predominant in phylum, Bacteroidetes, whereas genus, Alloprevotella, was less than $1 \%$ in the LC group. Within phylum, Firmicutes, genera, Pseudobutyrivibrio and Faecalibacterium, were more than $5 \%$ in the control group. Lactobacillus, Pseudobutyrivibrio, Anaerovibrio and Megasphaera represented more than $5 \%$ in the antibiotic group. Lactobacillus, Pseudobutyrivibrio and Megasphaera represented more than $5 \%$ in the YC group. Pseudobutyrivibrio, Faecalibacterium and Megasphaera represented more than 5\% in the LC group. Within phylum, Proteobacteria, genera, Campylobacter, in the control and Escherichia- 
Shigella in the antibiotic group were more than $1 \%$ of total bacteria. The abundance of genus, Succinivibrio, in the control group was higher than in the other groups.

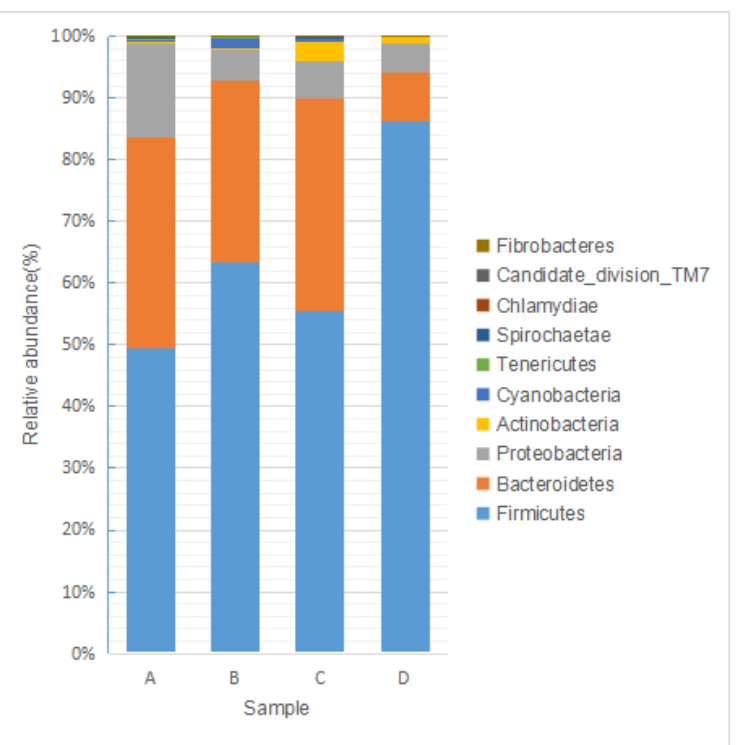

Figure 3 Microbial communities at phylum level

$\mathrm{A}=$ control group; $\mathrm{B}=$ antibiotic group; $\mathrm{C}=$ yeast culture group; and $\mathrm{D}=$ lactobacillus culture group

In the present study, regardless of the types of probiotics that were added to the diet, Firmicutes and Bacteroidetes were the dominant phyla in each group. These two phyla accounted for $>90 \%$ of total sequences in the antibiotic, YC and LC groups. However, Firmicutes and Bacteroidetes accounted for $84 \%$ of total sequences in the control group. Kim et al. (2011) showed similar results. Firmicutes and Bacteroidetes were the dominant phyla at various ages of pigs, and the increase of Firmicutes has been attributed to the growth of pigs (Zhao et al., 2015). It has been suggested that more Firmicutes and fewer Bacteroidetes is associated with an increased growth rate (Ban-Tokuda et al., 2017; Ley et al., 2005; Ley et al., 2006). In the previous study, the LC group had high levels of Firmicutes and low levels of Bacteroidetes, though the growth performance of the LC group was not the best (Du et al., 2017). Prevotella was the most predominant genus in all groups, except LC, and can promote the growth performance of pigs. It has also been reported that Megasphaera is the predominant bacteria in the faecal microbiota of weaning piglets (Mach et al., 2015; Slifierz et al., 2015). In this study, the results showed that Megasphaera accounted for the biggest proportion of total sequences in the LC group. Metabolites of Megasphaera such as vitamins, amino acids and short-chain fatty acids could provide the nutrients and energy for the host (Shetty et al., 2013), which could be the reason for increasing the growth performance in the LC group. The abundance of Campylobacter in the control group was more than $1 \%$, and it is considered a zoonotic pathogen (Tauxe, 2002; Graf et al., 2012). Lactobacillus in the YC and antibiotic groups was more abundant than in the control group. Increases in Lactobacillus improve the gut barrier by increasing the amount of mucin and blocking pathogens (Petri et al., 2010; Jacobi et al., 2012; Che et al., 2014). In addition, Lactobacillus can decrease the $\mathrm{pH}$ in the intestinal tract and inhibit the growth of harmful bacteria (Kim et al., 2012). Only the abundance of Shigella in the antibiotic group was represented by more than $1 \%$ of the total sequences compared with the other groups. This is supported by Janczyk et al. (2007) and Looft et al. (2014), who showed that antibiotics increase Shigella and Escherichia. For the YC group, mannose and glucans, the main components of yeast cell walls, could remove pathogens from the intestinal wall by agglutinating them (Oyofo et al., 1989; Spring et al., 2000; Ferket et al., 2002; Bovera et al., 2012).

The orthogonal partial least squares discriminate analysis (OPLS-DA) plots of the serum and caecum metabolomic data showed a clear separation between the control and antibiotic, control and YC, and control and LC groups (Figures 4 and 5). The serum metabolites were involved in three KEGG pathways, namely carbohydrate metabolism, lipid metabolism, and xenobiotics biodegradation and metabolism (Table 3). Compared with the control group, D-mannose, hexadecanoic acid, propanoic acid and a-D-glucopyranoside in the antibiotic group were enriched, while D-fructose in the antibiotic and LC groups was lower $(P<0.05)$. Octadecanoic acid in the YC group, and a-D-glucopyranoside in the YC and LC groups, and 1,2,3propanetricarboxylic acid in the LC group were decreased $(P<0.05)$ compared with the control group. D- 
fructose and $\alpha$-D-glucopyranoside in the YC group were increased compared with the antibiotic and LC groups $(P<0.05)$.

Table 2 Microbial communities at genus level in various treatment groups of weaning piglets

\begin{tabular}{|c|c|c|c|c|c|}
\hline Phyla & Genera & Control & Antibiotic & YC & LC \\
\hline \multirow{4}{*}{ Bacteroidetes } & Alloprevotella & 6.87 & 3.56 & 5.12 & $<1.00$ \\
\hline & Prevotella & 20.57 & 19.50 & 19.05 & 3.28 \\
\hline & uncultured & 4.13 & 5.09 & 6.92 & 1.95 \\
\hline & uncultured bacterium & 1.00 & $<1.00$ & $<1.00$ & 1.17 \\
\hline \multirow[t]{10}{*}{ Cyanobacteria } & Other & $<1.00$ & 1.53 & $<1.00$ & $<1.00$ \\
\hline & Lactobacillus & 4.50 & 8.18 & 9.54 & 1.36 \\
\hline & Streptococcus & 2.35 & 1.90 & 4.55 & 1.20 \\
\hline & Clostridium_sensu_stricto_1 & $<1.00$ & $<1.00$ & $<1.00$ & 1.93 \\
\hline & Clostridium_sensu_stricto_6 & $<1.00$ & $<1.00$ & $<1.00$ & 1.08 \\
\hline & Other & 1.05 & $<1.00$ & 1.13 & $<1.00$ \\
\hline & Blautia & 1.15 & $<1.00$ & 1.94 & 2.67 \\
\hline & Incertae_Sedis & 2.10 & 2.49 & 2.61 & 2.49 \\
\hline & Pseudobutyrivibrio & 7.84 & 14.44 & 7.08 & 13.90 \\
\hline & uncultured & 2.60 & 2.33 & 2.36 & 3.86 \\
\hline \multirow[t]{11}{*}{ Firmicutes } & Faecalibacterium & 6.49 & 4.42 & 3.25 & 11.25 \\
\hline & Ruminococcus & 1.57 & 1.02 & 1.10 & $<1.00$ \\
\hline & Subdoligranulum & 1.82 & 1.92 & 1.24 & 4.18 \\
\hline & uncultured & 4.46 & 3.39 & 3.42 & 6.50 \\
\hline & Phascolarctobacterium & 1.95 & 1.74 & 2.51 & 1.05 \\
\hline & Anaerovibrio & 2.05 & 7.97 & 2.25 & 3.90 \\
\hline & Dialister & $<1.00$ & $<1.00$ & $<1.00$ & 2.12 \\
\hline & Megasphaera & 2.59 & 6.51 & 7.61 & 15.98 \\
\hline & Mitsuokella & $<1.00$ & $<1.00$ & $<1.00$ & 2.97 \\
\hline & Selenomonas & 1.06 & 1.78 & $<1.00$ & $<1.00$ \\
\hline & Campylobacter & 1.50 & $<1.00$ & $<1.00$ & $<1.00$ \\
\hline \multirow[t]{2}{*}{ Proteobacteria } & Succinivibrio & 12.54 & 2.86 & 5.16 & 4.14 \\
\hline & Shigella & $<1.00$ & 1.29 & $<1.00$ & $<1.00$ \\
\hline
\end{tabular}

YC: yeast culture; LC: Lactobacillus culture

In the serum, seven metabolites changed significantly. The levels of D-mannose and D-glucose were higher in the antibiotic group, while the level of D-fructose was lower compared with the control group. Dfructose is phosphorylated to F-6-P and mannose is converted into F-6-P. F-6-P and D-glucose and are important intermediates and produce energy by entering the TCA cycle and glycolysis (Hirahatake et al., 2011; Pilvi et al., 2008; Nie et al., 2015). Dietary supplementation with antibiotics increased the metabolites of hexadecanoic acid and propanoic acid, which could cause higher digestion of fat and provide energy for growing pigs. Xiao et al. (2012) showed that hexadecanoic acid in metabolites was increased with dietary supplementation by L-glutamine. The concentrations of other metabolite in the different treatments were also checked. The concentration of $\alpha$-D-glucopyranoside in the control group was higher than in the other groups. 
The pathways in which a-D-glucopyranoside is involved are still unclear, and further research is needed to explore the effects of various additives on serum metabolites.

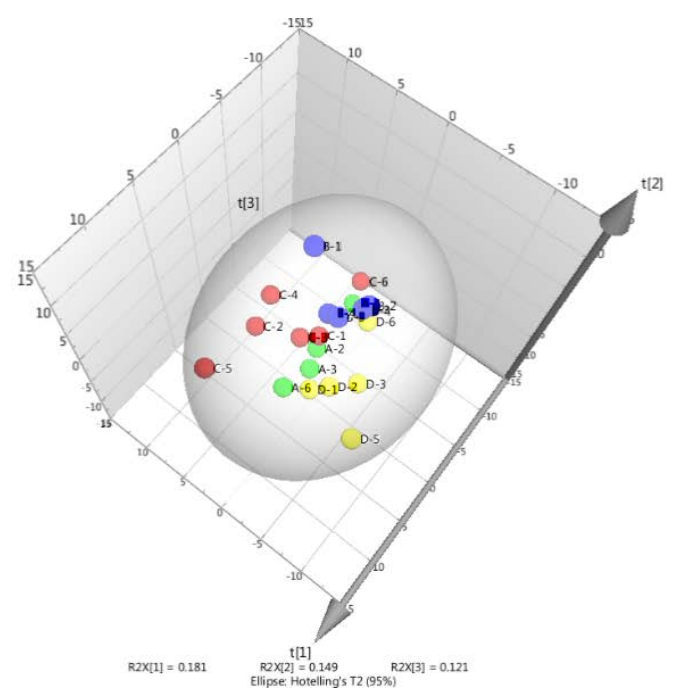

$\mathbf{H}_{-1}^{-1}$

Figure 4 Orthogonal partial least squares discriminate analysis plots of the gas chromatography mass spectrometry data for serum metabolites of the control versus antibiotic, control versus yeast culture, control versus lactobacillus culture, antibiotic versus yeast culture, and yeast culture versus lactobacillus culture groups

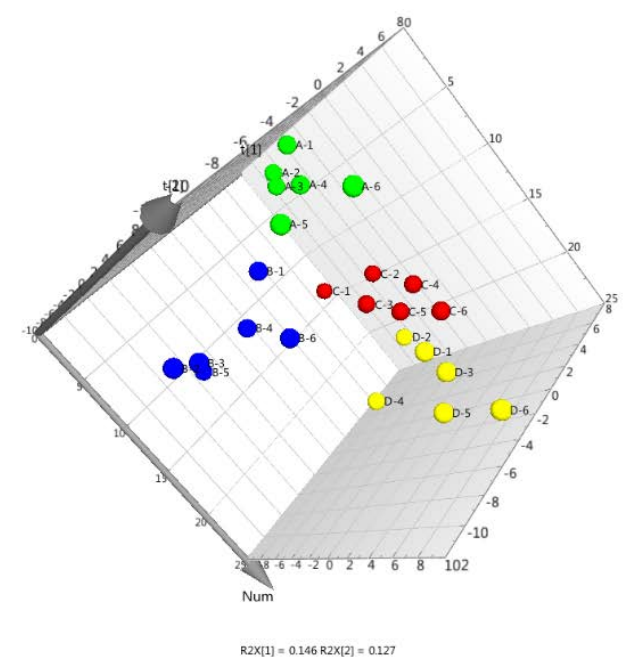

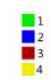

Figure 5 Orthogonal partial least squares discriminate analysis plots of the gas chromatography mass spectrometry data for the caecum metabolites of the control versus antibiotic, control versus lactobacillus culture, antibiotic versus yeast culture, antibiotic versus lactobacillus culture, and yeast culture versus lactobacillus culture groups

The caecum metabolites between control and antibiotic, control and LC, antibiotic and YC, and YC and LC groups were different (Table 4). Myo-inositol and benzenepropanoic acid concentrations in the antibiotic group were higher, while 11-trans-octadecenoic acid and butanoic acid were reduced in the LC group compared with the control $(P<0.05)$. Cis-9-hexadecenoic acid and heptadecanoic acid in the LC group were higher than in the control group $(P<0.05)$. D-mannose was richest in the LC group, followed by the antibiotic group, YC group, and the control group $(P<0.05)$. Compared with the antibiotic group, myo-inositol, D-fructose, L-arabinose, D-xylose, 9,12-octadecadienoic acid, and glucopyranose in the YC group, and cholesterol and cobaltocene in the LC group, were decreased $(P<0.05)$. Myo-inositol and 9,12octadecadienoic acids in the LC group were higher than in the YC group $(P<0.05)$. 
Table 3 Different serum metabolites of weaning piglets between the control, antibiotic, yeast culture and lactobacillus culture groups

\begin{tabular}{llcccc}
\hline Pathway & Metabolites & Groups & VIP & P-value & FC \\
\hline Carbohydrate metabolism & D-mannose & A vs B & 4.27 & 0.036 & 2.92 \\
& D-glucose & A vs B & 4.56 & 0.036 & 1.48 \\
& D-fructose & A vs B & 1.06 & 0.026 & 0.39 \\
& & A vs D & 2.21 & 0.006 & 0.22 \\
& & B vs C & 1.72 & 0.004 & 2.26 \\
Lipid metabolism & & C vs D & 1.64 & $<0.001$ & 0.26 \\
& Hexadecanoic acid & A vs B & 1.17 & 0.044 & 1.28 \\
Xenobiotics biodegradation and metabolism & Octadecanoic acid & A vs C & 1.34 & 0.019 & 0.80 \\
other & & B vs C & 1.72 & 0.002 & 0.79 \\
& Propanoic acid & A vs B & 3.68 & 0.021 & 1.45 \\
& a-D-glucopyranoside & A vs B & 2.03 & 0.007 & 0.15 \\
& & A vs C & 2.23 & 0.012 & 0.35 \\
& & A vs D & 3.47 & 0.005 & 0.19 \\
& & B vs C & 1.50 & 0.004 & 2.41 \\
& & C vs D & 1.03 & 0.050 & 0.54 \\
& & A vs D & 1.15 & 0.014 & 0.47 \\
\hline
\end{tabular}

A: control group, B: antibiotic group, C: yeast culture group and D: lactobacillus culture group

Table 4 Different caecum metabolites of weaning piglets among the control, antibiotic, yeast culture and lactobacillus culture groups

\begin{tabular}{|c|c|c|c|c|c|}
\hline Pathway & Metabolites & Groups & VIP & $P$-value & FC \\
\hline \multirow[t]{3}{*}{ Biosynthesis of other secondary metabolites } & Myo-inositol & $A$ vs $B$ & 2.39 & 0.021 & 2.80 \\
\hline & & B vs C & 2.53 & 0.004 & 0.20 \\
\hline & & C vs D & 1.56 & 0.052 & 2.94 \\
\hline \multirow[t]{7}{*}{ Carbohydrate metabolism } & D-mannose & A vs $B$ & 5.64 & 0.025 & 1.45 \\
\hline & & A vs D & 4.93 & 0.034 & 3.83 \\
\hline & & B vs C & 4.95 & 0.034 & 0.68 \\
\hline & & C vs D & 4.73 & 0.054 & 1.45 \\
\hline & D-fructose & B vs C & 3.49 & 0.008 & 0.31 \\
\hline & L-arbinose & B vs C & 1.80 & 0.016 & 0.53 \\
\hline & D-xylose & B vs C & 1.15 & 0.018 & 0.41 \\
\hline \multirow[t]{6}{*}{ Lipid metabolism } & Cholesterol & B vs D & 2.54 & 0.053 & 0.46 \\
\hline & 11-trans-octadecenoic acid & $A$ vs $B$ & 3.91 & 0.016 & 0.44 \\
\hline & cis-9-hexadecenoic acid & A vs D & 1.05 & 0.010 & 2.31 \\
\hline & cis-13-octadecenoic acid & B vs D & 4.58 & 0.021 & 2.54 \\
\hline & 9,12-octadecadienoic acid & C vs D & 3.79 & 0.032 & 1.48 \\
\hline & & B vs C & 3.42 & 0.022 & 0.72 \\
\hline Digestive system & Butanoic acid & $A$ vs $B$ & 1.06 & 0.011 & 0.40 \\
\hline \multirow[t]{4}{*}{ other } & Cobaltocene & B vs D & 1.38 & 0.037 & 0.26 \\
\hline & Glucopyranose & B vs C & 2.16 & 0.035 & 0.56 \\
\hline & Benzenepropanoic acid & $A$ vs $B$ & 1.09 & 0.035 & 4.21 \\
\hline & Heptadecanoic acid & A vs D & 1.20 & 0.045 & 2.46 \\
\hline
\end{tabular}

A: control group, B: antibiotic group, C: yeast culture group and D: lactobacillus culture group 
A total of 15 metabolites were identified in the caecum. They are involved mainly in the biosynthesis of other secondary metabolites, carbohydrate metabolism, lipid metabolism, and the digestive system. Myoinositol as a vitamin can eliminate reactive oxygen species and improve immune function (Jiang et al., 2016; Kuang et al., 2012). The increase of myo-inositol in the caecum of the antibiotic group may improve the immune system in weaning piglets. The level of D-mannose in the caecum was increased with dietary supplementation of antibiotic and LC, which was consistent with the effect of antibiotic on D-mannose in serum. The cis-9-hexadecenoic acid in the LC group may lead to a higher ability to digest fat. The concentrations of benzenepropanoic acid and heptadecanoic acid in the antibiotic and LC groups were higher than those in the control group, and the authors hypothesize that the high levels of these metabolites can produce more energy for the maintenance and growth of weaning piglets.

\section{Conclusion}

This work demonstrated that YC and LC supplementation in the weaning diet can improve the abundance of beneficial bacteria, though the concentrations of some metabolites in the serum and caecum were decreased when compared with the control group. Therefore, dietary supplementation of YC or LC could be used instead of antibiotics as additives for weaning piglets.

\section{Acknowledgments}

The authors are grateful to JLAU-Borui Dairy Science and Technology R\&D Centre of Jilin Agricultural University and Jilin Province Science and Technology Transformative Project (20160301003NY) P.R. China for accessing laboratory facilities and financial support.

\section{Authors' Contributions}

$\mathrm{XC}$ and $\mathrm{YL}$ were in charge of designing, conducting and analysing the experiment. All other authors participated in writing, interpreting and editing the manuscript.

\section{Conflict of Interest Declaration}

The authors declare that there is no conflict of interest.

\section{References}

Ban-Tokuda, T., Maekawa, S., Miwa, T., Ohkawara, S. \& Matsui, H., 2017. Changes in faecal bacteria during fattening in finishing swine. Anaerobe 47, 188-193.

Bolger, A.M. \& Lohse, M., 2014. Usadel B. Trimmomatic: A flexible trimmer for Illumina sequence data. Bioinformatics 30 , 2114-2120.

Bovera, F., Lestingi, A., lannaccone, F., Tateo, A. \& Nizza, A., 2012. Use of dietary mannanoligosaccharides during rabbit fattening period: Effects on growth performance, feed nutrient digestibility, carcass traits, and meat quality. J. Anim. Sci. 90, 3858-3866.

Caporaso, J.G., Lauber, C.L., Walters, W.A., Berg-Lyons, D., Huntley, J., Fierer, N. \& Gormley, N., 2012. Ultra-highthroughput microbial community analysis on the Illumina HiSeq and MiSeq platforms. The ISME J. 6, 1621-1624.

Castanon, J.I.R., 2007. History of the use of antibiotic as growth promoters in European poultry feeds. Poult. Sci. 86, 2466-2471.

Che, L., Chen, H., Yu, B., He, J., Zheng, P., Mao, X. \& Chen, D., 2014. Long-term intake of pea fiber affects colonic barrier function, bacterial and transcriptional profile in pig model. Nutrition and Cancer 66, 388-399.

Dona, A.C., Kyriakides, M., Scott, F., Shephard, E.A., Varshavi, D., Veselkov, K. \& Everett J.R., 2016. A guide to the identification of metabolites in NMR-based metabonomics/metabolomics experiments. Comput. Struct. Biotec. J. 14, 135-153.

Du, H.M., Wang, X.D., Wang, X.L., Yang, P.P., Qin, G.X. \& Zhen, Y.G., 2017. Effects of different probiotic cultures on growth performance, immunological function and blood biochemical indexes of weaned piglets. Chinese J. Vet. Sci. 37, 1379-1384+1400 (in Chinese)

Edgar, R.C., 2010. Search and clustering orders of magnitude faster than BLAST. Bioinformatics 26, $2460-2461$.

Edgar, R.C., Haas, B.J., Clemente, J.C., Quince, C. \& Knight, R., 2011. UCHIME improves sensitivity and speed of chimera detection. Bioinformatics 27, 2194-2200.

Ferket, P.R., 2002. Use of oligosaccharides and gut modifiers as replacements for dietary antibiotics. Minnesota Nutrition Conference 63, 169-182.

Fouhse, J.M., Zijlstra, R.T. \& Willing, B.P., 2016. The role of gut microbiota in the health and disease of pigs. Anim. Frontiers 6, 30-36.

Giang, H.H., Viet, T.Q., Ogle, B. \& Lindberg, J.E., 2010. Growth performance, digestibility, gut environment and health status in weaned piglets fed a diet supplemented with potentially probiotic complexes of lactic acid bacteria. Livest. Sci. 129, 95-103.

Graf, T.H., Havelaar, A., Klein, G., Koutsoumanis, K. \& Noerrung, B., 2012. Scientific opinion on a review on the European Union summary reports on trends and sources zoonoses, zoonotic agents and food-borne outbreaks in 2009 and 2010 - specifically for the data on Salmonella, Campylobacter, verotoxigenic Escherichia coli, Listeria monocytogenes and foodborne outbreaks. EFSA J. 10, 2726. 
He, Q., Kong, X., Wu, G., Ren, P., Tang, H., Hao, F. \& Tang, Z., 2009. Metabolomic analysis of the response of growing pigs to dietary L-Arginine supplementation. Amin. Acids 37, 199-208.

Heo, J.M., Opapeju, F.O., Pluske, J.R., Kim, J.C., Hampson, D.J. \& Nyachoti, C.M., 2013. Gastrointestinal health and function in weaned pigs: A review of feeding strategies to control post-weaning diarrhoea without using in feed antimicrobial compounds. J. Anim. Physiol. An. N. 97, 207-237.

Hirahatake, K.M., Meissen, J.K., Fiehn, O. \& Adams, S.H., 2011. Comparative effects of fructose and glucose on lipogenic gene expression and intermediary metabolism in HepG2 liver cells. PloS ONE 6, e26583.

Huse, S.M., Dethlefsen, L., Huber, J.A., Welch, D.M., Relman, D.A. \& Sogin, M.L., 2008. Exploring microbial diversity and taxonomy using SSU rRNA hypervariable tag sequencing. PLoS Genet. 4, e1000255.

Jacobi, S.K. \& Odle, J., 2012. Nutritional factors influencing intestinal health of the neonate. Adv. Nutr. 3, 687-696.

Janczyk, P., Pieper, R., Souffrant, W.B., Bimczok, D., Rothkötter, H.J. \& Smidt, H., 2007. Parenteral long-acting amoxicillin reduces intestinal bacterial community diversity in piglets even 5 weeks after the administration. The ISME J. 1, 180-183.

Jiang, W.D., Hu, K., Liu, Y., Jiang, J., Wu, P., Zhao, J. \& Feng, L., 2016. Dietary myo-inositol modulates immunity through antioxidant activity and the Nrf2 and E2F4/cyclin signalling factors in the head kidney and spleen following infection of juvenile fish with Aeromonas hydrophila. Fish Shellfish Immun. 49, 374-386.

Jiang, Z., Wei, S., Wang, Z., Zhu, C., Hu, S., Zheng, C. \& Yang, X., 2015. Effects of different forms of yeast Saccharomyces cerevisiae on growth performance, intestinal development, and systemic immunity in earlyweaned piglets. J. Anim. Sci. Biotechnol. 6, 47.

Kim, H.B., Borewicz, K., White, B.A., Singer, R.S., Sreevatsan, S., Tu, Z.J. \& Isaacson, R.E., 2011. Longitudinal investigation of the age-related bacterial diversity in the feces of commercial pigs. Vet. Microbiol. 153, 124-133.

Kim, J.C., Hansen, C.F., Mullan, B.P. \& Pluske, J.R., 2012. Nutrition and pathology of weaner pigs: Nutritional strategies to support barrier function in the gastrointestinal tract. Anim. Feed. Sci. Technol. 173, 3-16.

Kuang, S.Y., Xiao, W.W., Feng, L., Liu, Y., Jiang, J., Jiang, W.D. \& Zhou, X.Q., 2012. Effects of graded levels of dietary methionine hydroxy analogue on immune response and antioxidant status of immune organs in juvenile Jian carp (Cyprinus carpio var. Jian). Fish Shellfish Immun. 32, 629-636.

Lan, R.X., Koo, J.M. \& Kim, I.H., 2016. Effects of Lactobacillus acidophilus supplementation in different energy and nutrient density diets on growth performance, nutrient digestibility, blood characteristics, fecal microbiota shedding, and fecal noxious gas emission in weaning pigs. Anim. Feed Sci. Technol. 219, 181-188.

Lan, Y., Verstegen, M.W.A., Tamminga, S. \& Williams, B.A., 2005. The role of the commensal gut microbial community in broiler chickens. Wrld's Poult. Sci. J. 61, 95-104.

Ley, R.E., Bäckhed, F., Turnbaugh, P., Lozupone, C.A. \& Knight, R.D., Gordon, J.I., 2005. Obesity alters gut microbial ecology. P. Natl. Acad. Sci. USA. 102, 11070-11075.

Ley, R.E., Turnbaugh, P.J., Klein, S. \& Gordon, J.I., 2006. Microbial ecology: Human gut microbes associated with obesity. Nature 444, 1022-1023.

Looft, T., Allen, H.K., Cantarel, B.L., Levine, U.Y., Bayles, D.O., Alt, D.P. \& Stanton, T.B., 2014. Bacteria, phages and pigs: the effects of in-feed antibiotics on the microbiome at different gut locations. The ISME J. 8, 1566-1576.

Mach, N., Berri, M., Estellé, J., Levenez, F., Lemonnier, G., Denis, C. \& Rogel-Gaillard, C., 2015. Early life establishment of the swine gut microbiome and impact on host phenotypes. Env. Microbiol. Rep. 7, 554-569.

Magoč, T. \& Salzberg, S.L., 2011. FLASH: Fast length adjustment of short reads to improve genome assemblies. Bioinformatics 27, 2957-2963.

Nie, C.X., Zhang, W.J., Wang, Y.Q., Liu, Y.F., Ge, W.X. \& Liu, J.C., 2015. Tissue lipid metabolism and hepatic metabolomic profiling in response to supplementation of fermented cottonseed meal in the diets of broiler chickens. J. Zhejiang Univ. Sci. b. 16, 447-455.

Niederwerder, M.C., 2017. Role of the microbiome in swine respiratory disease. Vet. Microbiol. 209, 97-106.

NRC, 2012. Nutrient Requirements of Swine. National Academies Press. Washington DC, USA.

Oyofo, B.A., DeLoach, J.R., Corrier, D.E., Norman, J.O., Ziprin, R.L. \& Mollenhauer, H.H., 1989. Prevention of Salmonella typhimurium colonization of broilers with D-mannose. Poult. Sci. 68, 1357-1360.

Petri, D., Hill, J.E. \& Van Kessel, A.G., 2010. Microbial succession in the gastrointestinal tract (GIT) of the preweaned pig. Livest. Sci 133, 107-109.

Pilvi, T.K., Seppänen-Laakso, T., Simolin, H., Finckenberg, P., Huotari, A., Herzig, K.H. \& Mervaala, E.M., 2008. Metabolomic changes in fatty liver can be modified by dietary protein and calcium during energy restriction. Wrld J. Gastroentero (WJG). 14, 4462.

Sekirov, I., Tam, N.M., Jogova, M., Robertson, M.L., Li, Y., Lupp, C. \& Finlay, B.B., 2008. Antibiotic-induced perturbations of the intestinal microbiota alter host susceptibility to enteric infection. Infect. Immun. 76, 4726-4736.

Shetty, S.A., Marathe, N.P., Lanjekar, V., Ranade, D. \& Shouche, Y.S., 2013. Comparative genome analysis of Megasphaera sp. reveals niche specialization and its potential role in the human gut. PloS ONE 8, e79353.

Slifierz, M.J., Friendship, R.M. \& Weese, J.S., 2015. Longitudinal study of the early-life fecal and nasal microbiotas of the domestic pig. BMC Microbiol. 15, 184.

Spring, P., Wenk, C., Dawson, K.A. \& Newman, K.E., 2000. The effects of dietary mannaoligosaccharides on cecal parameters and the concentrations of enteric bacteria in the ceca of salmonella-challenged broiler chicks. Poult. Sci. 79, 205-211.

Tauxe, R.V., 2002. Emerging foodborne pathogens. Int. J. Food Microbiol. 78, 31-41.

Torrallardona, D., Conde, R., Badiola, I. \& Polo, J., 2007. Evaluation of spray dried animal plasma and calcium formate as alternatives to colistin in piglets experimentally infected with Escherichia coli K99. Livest. Sci.108, 303-306. 
Weedman, S.M., Rostagno, M.H., Patterson, J.A., Yoon, I., Fitzner, G. \& Eicher, S.D., 2011. Yeast culture supplement during nursing and transport affects immunity and intestinal microbial ecology of weanling pigs. J. Anim. Sci. 89, 1908-1921.

Williams, N.H., Stahly, T.S. \& Zimmerman, D.R., 1997. Effect of chronic immune system activation on the rate, efficiency, and composition of growth and lysine needs of pigs fed from 6 to $27 \mathrm{~kg}$. J. Anim. Sci. 75, 2463-2471.

Xiao, Y.P., Wu, T.X., Hong, Q.H., Sun, J.M., Chen, A.G., Yang, C.M. \& Li, X.Y., 2012. Response to weaning and dietary L-glutamine supplementation: Metabolomic analysis in piglets by gas chromatography/mass spectrometry. J. Zhejiang Univ. Sci. b. 13, 567-578.

Xiong, X., Yang, H., Li, B., Liu, G., Huang, R., Li, F. \& Deng, D., 2015. Dietary supplementation with yeast product improves intestinal function, and serum and ileal amino acid contents in weaned piglets. Livest. Sci. 171, $20-27$.

Zhao, W., Wang, Y., Liu, S., Huang, J., Zhai, Z., He, C. \& Zhao, J., 2015. The dynamic distribution of porcine microbiota across different ages and gastrointestinal tract segments. PloS ONE 10, e0117441.

Zhou, L., Fang, L., Sun, Y., Su, Y. \& Zhu, W., 2016. Effects of the dietary protein level on the microbial composition and metabolomic profile in the hindgut of the pig. Anaerobe $38,61-69$. 